\title{
Ventricular Repolarization Lability in Children With Kawasaki Disease
}

\author{
Marina Kuriki $\cdot$ Masayuki Fujino $\cdot$ Ken-ichi Tanaka \\ Kayo Horio · Hirofumi Kusuki • Misa Hosoi • Yoshihiko Eryu • \\ Tomochika Kato $\cdot$ Toshio Yamazaki $\cdot$ Tadayoshi Hata
}

Received: 31 October 2010/ Accepted: 31 January 2011/Published online: 23 February 2011

(c) The Author(s) 2011. This article is published with open access at Springerlink.com

\begin{abstract}
Kawasaki disease (KD) is an acute febrile disease of unknown etiology that develops in children and is sometimes accompanied by myocardial dysfunction and systemic vasculitis. However, myocardial repolarization lability has not yet been fully investigated. Thus, the objective of this study was to evaluate myocardial repolarization lability (QT variability index-QTVI) based on the body surface electrocardiograms in the acute and recovery phases. The subjects were 25 children with acute $\mathrm{KD}$ who were hospitalized for treatment. An equal number of age-matched healthy children were selected as controls.The RR-intervals and QT-intervals were measured based on a body surface electrocardiogram of 120 consecutive heartbeats to calculate the QTVI. The QTVI values were then compared with the acute and recovery phases. The relationships between blood biochemistry data and QTVI values were also examined. QTVI was significantly
\end{abstract}

\footnotetext{
M. Kuriki · K. Horio · T. Hata ( $\square)$

Graduate School of Health Sciences, Fujita Health University, Toyoake, Japan

e-mail: thata@fujita-hu.ac.jp

M. Fujino · Y. Eryu · T. Yamazaki

Department of Pediatrics, Fujita Health University,

Toyoake, Japan

K. Tanaka $\cdot$ T. Kato

Department of Pediatrics, Toyokawa Municipal Hospital,

Toyokawa, Japan

H. Kusuki

Clinical Laboratory, Mie University Hospital, Tsu, Japan

M. Hosoi

Clinical Laboratory, Fujita Health University Hospital,

Toyoake, Japan
}

decreased from the acute phase to the recovery phase $(P<0.05)$ and then recovered to the same level as that of the control. QTVI in the acute phase showed a significant positive relationship with body temperature and C-reactive protein $(P<0.05)$. QTVI was high in the acute phase and was correlated with an inflammatory reaction and became normalized during the recovery phase.

Keywords Kawasaki disease - Ventricular repolarization instability · QT interval variability

\section{Introduction}

Kawasaki disease (KD) is an acute febrile disease of unknown etiology that develops in children. Recently, the incidence of KD has reached $>10,000$ cases/year in Japan. There have been some cases of systemic vasculitis and myocardial dysfunction as a consequence of this disease. Dilatation of the coronary artery has also been observed, although the incidence is low. It has been shown that instability of myocardial repolarization is increased because of ischemic myocardial failure in patients with coronary arterial aneurysm as complications $[1,2]$. Therefore, the evaluation of myocardial failure, including proarrhythmic substrate in the acute phase, could be important for understanding the pathophysiology of KD. To date, no comparative study on alterations of myocardial repolarization from the acute phase to the recovery phase of KD has been reported. Thus, the lability of myocardial repolarization accompanied by inflammation remains unknown. The objectives of this study were to compare the QT variability index (QTVI) [3] in the acute phase of KD and in the recovery phase after treatment with immunoglobulin based on a body surface ECG. We also investigated correlations 
between QTVI values and blood biochemistry data and evaluated the instability of myocardial repolarization.

\section{Subjects}

The subjects were 30 children with KD who were hospitalized at Fujita Health University Hospital and Toyokawa Municipal Hospital. After the diagnosis was made, all patients had fulfilled the American Heart Association guidelines for diagnosis of $\mathrm{KD}$ and received intravenous immunoglobulin $(2 \mathrm{~g} / \mathrm{kg})$ treatment $(5.2 \pm 0.8$ days after the onset of fever). In addition, aspirin was given at a dose of $50 \mathrm{mg} / \mathrm{kg} / \mathrm{d}$ for the first 5-10 days and was followed by a lower antiplatelet dose $(5 \mathrm{mg} / \mathrm{kg})$ for the next 4-6 weeks. For the present analysis, three patients were excluded because stable electrocardiogram (ECG) recordings were not possible due to vigorous motion and crying. An additional two patients were excluded because they showed extremely high heart rates (HRs; > $180 \mathrm{bpm}$ ) with overlapping of T-wave terminal portion and $\mathrm{P}$ wave. Finally, 25 patients (16 boys and 9 girls; age $2.2 \pm 2.1$ years) were included in this study. The average febrile period was $7.5 \pm 2.5$ days; the average body temperature was $37.9^{\circ} \mathrm{C} \pm 1.1^{\circ} \mathrm{C}$ at the time of ECG the acute phase; and the average hospitalization period was $15.5 \pm 5.0$ days. The $\mathrm{Z}$ score ( $\mathrm{SD} \geq+2.5$ was determined to be significant) [4] was used for the evaluation of coronary artery ectasia. Although transient coronary artery abnormalities were observed during the course of the disease in four patients, all patients were discharged from hospital with no complications, including coronary artery aneurysm. An equal number of healthy children (age $2.2 \pm 0.4$ years), who were age- and sex-matched comparably to the subjects, were used as controls. This study was approved by our institution's Ethics Committee.

\section{Methods}

After obtaining written informed consent from parents, electrocardiography (CM5-lead) was conducted during cardiac ultrasonography using a biopolygraph MP-150 (Biopac Systems, Goleta, CA). Pretreatment with sodium trichloroethyl phosphate syrup $(0.7 \mathrm{ml} / \mathrm{kg})$ was conducted for tranquilization in children age 3-12 months. The RRinterval was automatically recorded based on the original electrocardiographic data of 120 consecutive heartbeats (which showed no arrhythmia and no overlapping of the terminal portion of the T-wave and the ascending limb of the P-wave) using Acqknowledge version 3.9 (Biopac Systems). The starting point of the $\mathrm{Q}$ waves and the end of the $\mathrm{T}$ waves were determined by linear derivation and the absolute values to measure the RR- and QT-intervals of the same heartbeats. The instantaneous HR was determined based on the measured RR-intervals to calculate the mean $\mathrm{HR}(\mathrm{HRm})$ and variance of HR (HRv) of the 120 heartbeats. The mean QT-interval (QTm) and variance of the QT-interval (QTV) were determined based on the QT-intervals of the same heartbeats to compare the QTVI in the acute $(7.2 \pm 0.7$ days $)$ and recovery $(22.0 \pm 4.1$ days $)$ phases using the formula described by Berger et al. [3] (Eq. 1):

$\mathrm{QTVI}=\log _{10}\left\{\left(\mathrm{QTv} / \mathrm{QTm}^{2}\right) /\left(\mathrm{HRv} / \mathrm{HRm}^{2}\right)\right\}$.

The relationships between blood biochemistry data at the time of hospitalization and QTVI values and those between body temperature and QTVI values were analyzed. Serum chemistry examinations were conducted for white blood cell count (WBC), neutrophils, hematocrit, platelets, C-reactive protein (CRP), total protein, albumin, sodium, potassium, chloride, aspartate transaminase (AST), alanine transaminase (ALT), and total bilirubin. None of the patients required treatment, such as administration of catecholamine, diuretics, etc., for heart failure, and no agents that might have affected the QT-interval were administered. For statistical analysis, JMP statistical analysis software version 5.1.2 (SAS Cary, NC) was used. Paired Student $t$ test was conducted to compare the blood biochemistry data and ECG parameters of acute and recovery phases, and Pearson's correlation coefficients analysis was conducted for CRP, body temperature and QTVI in acute phase. Tukey-Kramer Honestly Significant Difference test was used to compare the QTVI of KD patients with that in healthy children. A $p$ value $<0.05$ was considered to be significant.

\section{Results}

\section{Comparison of ECG Parameters}

No significant differences were found in mean RR-interval, variance of RR-interval, and variance of $H R$, whereas significant differences were observed in the variance of QT-interval between the acute and recovery phases (Table 1).

Comparison of QTVI Values in the Acute and Recovery Phases

QTVI was significantly decreased from the acute phase to the recovery phase $(-0.60 \pm 0.54$ to $-1.22 \pm 0.31, p<0.05)$ and was then restored to a value equivalent to that of the healthy controls (Fig. 1). Figure 2 shows typical ECG patterns from a patient in the acute and recovery phases. 
Table 1 Comparison of ECG parameters in the acute and recovery phases

\begin{tabular}{lccl}
\hline Parameters & Acute phase & Recovery phase & $p$ \\
\hline RRm (ms) & $525.3 \pm 97.6$ & $530.8 \pm 53.8$ & 0.770 \\
RRv (ms) & $940.3 \pm 471.2$ & $721.6 \pm 502.1$ & 0.473 \\
HRm (ms) & $118.4 \pm 22.1$ & $114.4 \pm 11.4$ & 0.355 \\
HRv (ms) & $31.2 \pm 40.2$ & $32.9 \pm 26.7$ & 0.803 \\
QTm (ms) & $301.2 \pm 39.3$ & $304.8 \pm 23.9$ & 0.593 \\
QTv (ms) & $39.5 \pm 46.3^{\mathrm{a}}$ & $13.0 \pm 7.8$ & $<0.05$ \\
QTcB (ms) & $416.6 \pm 26.6$ & $419.2 \pm 23.7$ & 0.598 \\
QTcF (ms) & $375.3 \pm 29.9$ & $376.8 \pm 22.6$ & 0.763 \\
QTVI & $-0.60 \pm 0.54^{\mathrm{a}}$ & $-1.22 \pm 0.31$ & $<0.05$ \\
\hline
\end{tabular}

QTcB = Corrected QT Interval by Bazett formula; QTcF = Corrected QT Interval by Fridericia formula

${ }^{\text {a }}$ Value expressed as the mean $\pm \mathrm{SD}(p<0.05)$

\begin{tabular}{|c|}
\multicolumn{1}{c|}{$p<0.05$} \\
\hline$p<0.05 \quad$ N.S. \\
\hline
\end{tabular}

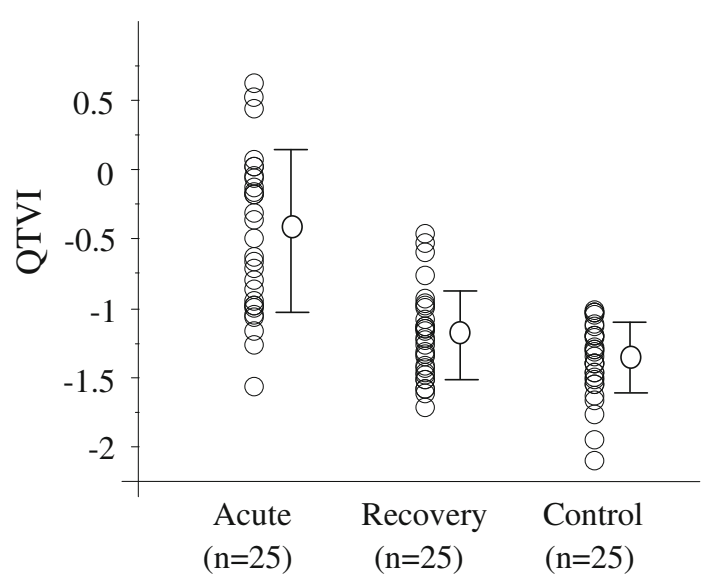

Fig. 1 Comparison of QTVI values in the acute and recovery phases

\section{Blood Biochemistry Data and QTVI Values}

There were significant differences in WBC, hematocrit, sodium, total protein, albumin, and CRP between the acute and recovery phases (Table 2). There were no significant differences in platelets, potassium, chloride, total bilirubin, AST, and ALT between the acute and recovery phases. Pearson's correlation coefficients of CPR and QTVI values in the acute phase showed a significant positive correlation $(r=0.404$; Fig. 3).

\section{Body Temperature and QTVI Values}

Pearson's correlation coefficients of body temperature and QTVI values in the acute phase showed a significant positive correlation ( $r=0.607$; Fig. 4$)$, and QTVI increased in accordance with increased body temperature.

\section{Discussion}

The etiology of KD has not been clarified to date, $>40$ years after its discovery. However, with regard to the etiology of this disease, overproduction of inflammatory cytokines is believed to cause myocarditis or a lesion in the coronary artery $[5,6]$. However, sufficient clarification of the electrophysiological changes due to inflammation in the acute phase has not yet been achieved. Thus, in the current study, we calculated QTVI based on a noninvasive body surface ECG to assess abnormal myocardial repolarization in the acute phase.

The QTVI used in this study is a new index reflecting instability of repolarization of the ventricular myocardium, which is calculated based on a single-lead body surface ECG and represents the log ratio between the QT interval and HR variability, each normalized by the squared mean of process. This index has been reported to be high in patients with dilated cardiomyopathy $[7,8]$, in those at risk for fatal arrythmias, and in those with hypertrophic cardiomyopathy [9].

In this study, we found that QTVI was significantly increased in the acute phase compared with the recovery phase. Furthermore, QTVI was restored to the same level as that of healthy controls in the recovery phase. With regard to the relationship between body temperature and blood biochemistry data in our patients, QTVI index had a significant positive correlation with body temperature and CRP. Based on these findings, we speculated that the onset of fever caused by the release of various cytokines and CRP, which is closely related to interleukin (IL)-6, strongly affected myocardial repolarization in the acute phase of $\operatorname{KD}[5,6]$.

Because HR generally increases in accordance with the onset of fever, catecholamines are considered to affect HR directly and QTVI is thought to be increased by augmented sympathetic nerve activity in patients with heart failure [10]. However, recent findings have shown that there is no correlation between serum concentrations of norepinephrine, which activates the sympathetic nervous system, and QTVI in patients without heart failure; [11] i.e., the subjects were children without heart failure, and the increased QTVI observed in the acute phase was not considered to depend on sympathetic tone caused by the onset of fever but rather on the inflammatory humoral regulators described previously. Similarly, increased cytokines such as tumor necrosis factor-alpha and IL-6, which cause systemic inflammation, have been reported in the acute phase of KD $[12,13]$. Furthermore, recent reports of in vitro studies have indicated alterations in ionic currents by cytokines, 
Fig. 2 Comparison of QTVI values between acute and recovery phases in a typical case. Four to 6 of the 120 measured heartbeats are shown as representative heartbeats
Acute phase

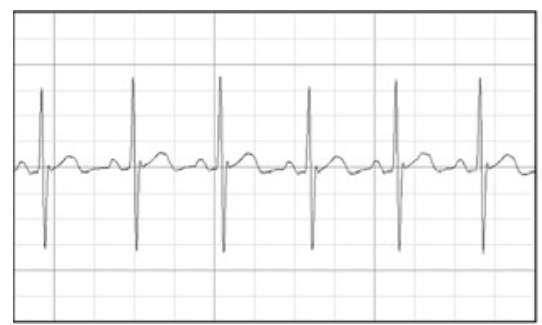

QTVI=0.06

$\left(\begin{array}{ll}\mathrm{HRm}=113.8 & \mathrm{HRv}=3.5 \\ \mathrm{QTm}=295.2 & \mathrm{QTv}=26.9\end{array}\right)$
Recovery phase

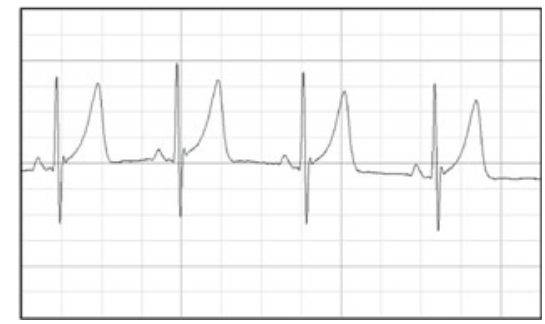

QTVI=-1.49

$\left(\begin{array}{cc}\mathrm{HRm}=95.3 & \mathrm{HRv}=26.7 \\ \mathrm{QTm}=342.3 & \mathrm{QTv}=11.2\end{array}\right)$
Table 2 Comparison of blood biochemistry data in the acute and recovery phases

\begin{tabular}{lccl}
\hline Biochemistry data & Acute phase & Recovery phase & $p$ \\
\hline WBC $\left(10^{2} / \mathrm{mm}^{3}\right)$ & $127.3 \pm 57.3^{\mathrm{a}}$ & $83.9 \pm 34.5$ & $<0.05$ \\
Hematocrit $(\%)$ & $32.1 \pm 4.0^{\mathrm{a}}$ & $34.4 \pm 2.9$ & $<0.05$ \\
Platelet $\left(10^{4} / \mathrm{mm}^{3}\right)$ & $42.0 \pm 17.1$ & $44.3 \pm 20.6$ & 0.672 \\
Total bilirubin $(\mathrm{mg} / \mathrm{dl})$ & $0.5 \pm 0.4$ & $0.4 \pm 0.1$ & 0.508 \\
AST $(\mathrm{IU} / \mathrm{l})$ & $40.0 \pm 25.9$ & $40.0 \pm 17.2$ & 0.995 \\
ALT $(\mathrm{IU} / \mathrm{l})$ & $28.4 \pm 26.8$ & $19.0 \pm 13.0$ & 0.132 \\
Sodium $(\mathrm{mmol} / \mathrm{l})$ & $134.7 \pm 2.9^{\mathrm{a}}$ & $137.2 \pm 1.7$ & $<0.05$ \\
Potassium $(\mathrm{mmol} / \mathrm{l})$ & $4.7 \pm 0.6$ & $4.6 \pm 0.5$ & 0.295 \\
Chloride $(\mathrm{mmol} / \mathrm{l})$ & $99.4 \pm 4.0^{\mathrm{a}}$ & $101.8 \pm 1.2$ & $<0.05$ \\
Total protein $(\mathrm{g} / \mathrm{dl})$ & $7.5 \pm 1.1^{\mathrm{a}}$ & $8.1 \pm 0.7$ & $<0.05$ \\
Albumin $(\mathrm{g} / \mathrm{dl})$ & $3.3 \pm 0.6^{\mathrm{a}}$ & $4.0 \pm 0.4$ & $<0.05$ \\
CRP $(\mathrm{mg} / \mathrm{dl})$ & $5.7 \pm 3.6^{\mathrm{a}}$ & $0.3 \pm 0.1$ & $<0.05$ \\
\hline
\end{tabular}

${ }^{a}$ Value expressed as mean \pm SD $(p<0.05)$

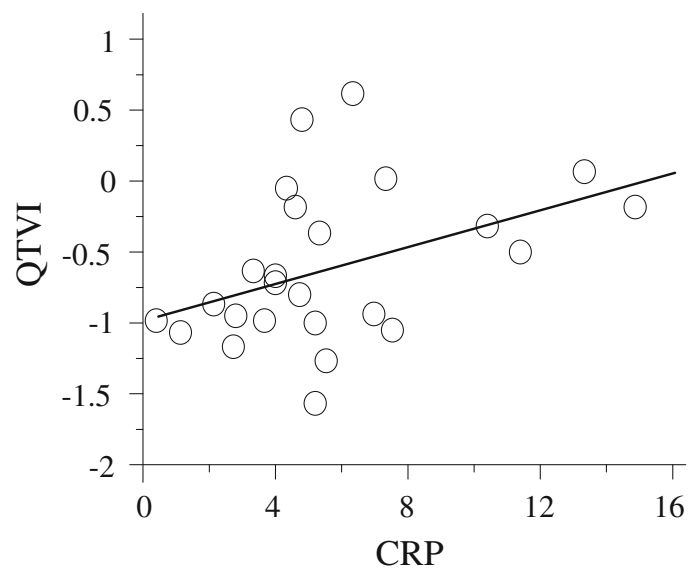

Fig. 3 CRP versus QTVI. CRP and QTVI values in the acute phase showed a significant positive linear correlation $(r=0.404$, $p=0.045$ )

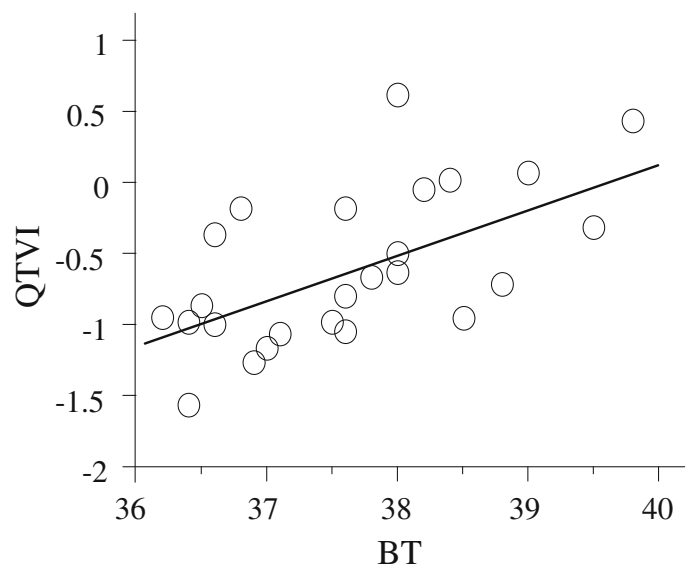

Fig. 4 Body temperature $\left({ }^{\circ} \mathrm{C}\right)$ versus QTVI. Body temperature and QTVI showed a significant positive correlation in the acute phase $(r=0.607, p=0.0001)$

which are responsible for myocardial action potentials $[14,15]$. Based on the results of our study, we also believe that myocardial repolarization was altered by humoral regulators, such as cytokines, in the febrile phase, when an inflammatory reaction was strong in children affected with KD. Only sporadic premature ventricular contractions were observed, and no serious arrhythmias, such as feverinduced ventricular tachycardia [16], were observed in this study, but these arrhythmias may be related not only to a prolonged myocardial repolarization time but also instability of the ionic current and the refractory period of action potential $[17,18]$. However, QTVI can stratify the risk of arrhythmogenesis caused by inflammation even when there is no cardiac channelopathy, and electrophysiological evaluation of the myocardium using this method is considered to be useful for patients such as children, in whom invasive examinations including coronary angiography, exercise stress, and myocardial scintigram are restricted. Further prospective larger-scale studies are 
needed to prove that QTVI can be a predictor of lethal arrhythmias.

\section{Study Limitations}

Using the current methods, it was difficult to analyze the QT-interval automatically, based on the record of overlapping the terminal portion of the $T$ wave and the ascending limb of the $\mathrm{P}$ wave when the HR s exceeded $180 \mathrm{bpm}$.

\section{Conclusion}

We conducted a comparative investigation of the characteristics of myocardial repolarization in the acute and recovery phases of KD based on QTVI values. We showed that QTVI was high and that the lability of myocardial repolarization was increased in the acute phase, indicating the involvement of humoral regulators, which were reflected by the fever and CRP. Although arrythmogenesis may not be evident, subclinical ventricular repolarization lability may be present (and is independent of coronary artery involvement). Further studies may be warranted to clarify the mechanisms underlying ventricular repolarization lability and elucidate the relationships between arrhythmogenesity and inflammation of KD.

Open Access This article is distributed under the terms of the Creative Commons Attribution Noncommercial License which permits any noncommercial use, distribution, and reproduction in any medium, provided the original author(s) and source are credited.

\section{References}

1. Osada M, Tanaka Y, Komai T, Maeda Y, Kitano M, Komori S et al (1999) Coronary arterial involvement and QT dispersion in Kawasaki disease. Am J Cardiol 84:466-468

2. Dahdah NS, Jaeggi E, Fournier A (2002) Electrocardiographic depolarization and repolarization: long-term after Kawasaki disease. Pediatr Cardiol 23:513-517

3. Berger RD, Kasper EK, Baughman KL, Marban E, Calkins H, Tomaselli GF (1997) Beat-to-beat QT interval variability: novel evidence for repolarization lability in ischemic and nonischemic dilated cardiomyopathy. Circulation 96:1557-1565
4. McCrindle BW, Li JS, Minich LL, Colan SD, Atz AM, Takahashi $M$ et al (2007) Coronary artery involvement in children with Kawasaki disease: risk factors from analysis of serial normalized measurements. Circulation 116:174-179

5. Lin CY, Lin CC, Hwang B, Chiang B (1992) Serial changes of serum interleukin-6, interleukin-8, and tumor necrosis factor alpha among patients with Kawasaki disease. J Pediatr 121: 924-926

6. Lin CY, Lin CC, Hwang B, Chiang BN (1993) Cytokines predict coronary aneurysm formation in Kawasaki disease patients. Eur J Pediatr 152:309-312

7. Berger RD (2003) QT variability. J Electrocardiol 36:83-87

8. Atiga WL, Calkins H, Lawrence JH, Tomaselli GF, Smith JM, Berger RD (1998) Beat-to-beat repolarization lability identifies patients at risk for sudden cardiac death. J Cardiovasc Electrophysiol 9:899-908

9. Atiga WL, Fananapazir L, McAreavey D, Calkins H, Berger RD (2000) Temporal repolarization lability in hypertrophic cardiomyopathy caused by beta-myosin heavy-chain gene mutations. Circulation 101:1237-1242

10. Desai N, Raghunandan DS, Mallavarapu M, Berger RD, Yeragani VK (2004) Beat-to-beat heart rate and QT variability in patients with congestive cardiac failure: blunted response to orthostatic challenge. Ann Noninvasive Electrocardiol 9:323-329

11. Baumert M, Lambert GW, Dawood T, Lambert EA, Esler MD, McGrane $M$ et al (2008) QT interval variability and cardiac norepinephrine spillover in patients with depression and panic disorder. Am J Physiol Heart Circ Physiol 295:962-968

12. Quasney MW, Bronstein DE, Cantor RM, Zhang Q, Stroupe C, Shike H et al (2001) Increased frequency of alleles associated with elevated tumor necrosis factor-alpha levels in children with Kawasaki disease. Pediatr Res 49:686-690

13. Suganami Y, Kawashima H, Hasegawa D, Sato S, Hoshika A (2008) Clinical application of rapid assay of serum interleukin-6 in Kawasaki disease. Pediatr Int 50:264-266

14. Kawada H, Niwano S, Niwano H, Yumoto Y, Wakisaka Y, Yuge $M$ et al (2006) Tumor necrosis factor-alpha downregulates the voltage gated outward $\mathrm{K}+$ current in cultured neonatal rat cardiomyocytes: a possible cause of electrical remodeling in diseased hearts. Circ J 70:605-609

15. Ino K, Watanabe H, Saito T, Kibira S, Iijima T, Miura M (2003) TNF-alpha rapidly antagonizes the beta-adrenergic responses of the chloride current in guinea-pig ventricular myocytes. Circ J 67: 347-353

16. Amin AS, Herfst LJ, Delisle BP, Klemens CA, Rook MB, Bezzina CR et al (2008) Fever-induced QTc prolongation and ventricular arrhythmias in individuals with type 2 congenital long QT syndrome. J Clin Invest 118:2552-2561

17. Day CP, McComb JM, Campbell RW (1990) QT dispersion: an indication of arrhythmia risk in patients with long QT intervals. Br Heart J 63:342-344

18. Gupta P, Patel C, Patel H, Narayanaswamy S, Malhotra B, Green JT et al (2008) T(p-e)/QT ratio as an index of arrhythmogenesis. J Electrocardiol 41:567-574 